\title{
Simulations of the atomic structure, energetics, and cross slip of screw dislocations in
} copper

Rasmussen, Torben; Jacobsen, Karsten Wedel; Leffers, Torben; Pedersen, Ole Bøcker

Published in:

Physical Review B

Link to article, DOI:

10.1103/PhysRevB.56.2977

Publication date:

1997

Document Version

Publisher's PDF, also known as Version of record

Link back to DTU Orbit

Citation (APA):

Rasmussen, T., Jacobsen, K. W., Leffers, T., \& Pedersen, O. B. (1997). Simulations of the atomic structure, energetics, and cross slip of screw dislocations in copper. Physical Review B, 56(6), 2977-2990.

https://doi.org/10.1103/PhysRevB.56.2977

\section{General rights}

Copyright and moral rights for the publications made accessible in the public portal are retained by the authors and/or other copyright owners and it is a condition of accessing publications that users recognise and abide by the legal requirements associated with these rights.

- Users may download and print one copy of any publication from the public portal for the purpose of private study or research.

- You may not further distribute the material or use it for any profit-making activity or commercial gain

- You may freely distribute the URL identifying the publication in the public portal 


\title{
Simulations of the atomic structure, energetics, and cross slip of screw dislocations in copper
}

\author{
T. Rasmussen \\ Center for Atomic-scale Materials Physics, Department of Physics, Technical University of Denmark, DK-2800 Lyngby, Denmark \\ and Materials Research Department, Risb National Laboratory, DK-4000 Roskilde, Denmark \\ K. W. Jacobsen \\ Center for Atomic-scale Materials Physics, Department of Physics, Technical University of Denmark, DK-2800 Lyngby, Denmark \\ T. Leffers and O. B. Pedersen \\ Materials Research Department, Risb National Laboratory, DK-4000 Roskilde, Denmark
}

(Received 12 December 1996)

\begin{abstract}
Using nanoscale atomistic simulations it has been possible to address the problem of cross slip of a dissociated screw dislocation in an fcc metal $(\mathrm{Cu})$ by a method not suffering from the limitations imposed by elasticity theory. The focus has been on different dislocation configurations relevant for cross slip via the Friedel-Escaig (FE) cross-slip mechanism. The stress free cross-slip activation energy and activation length for this mechanism are determined. We show that the two constrictions necessary for cross slip in the FE cross-slip mechanism are not equivalent and that a dislocation configuration with just one of these constrictions is energetically favored over two parallel Shockley partials. The effect of having the dislocation perpendicular to a free surface is investigated. The results are in qualitative agreement with transmission electron microscopy experiments and predictions from linear elasticity theory showing recombination or repulsion of the partials near the free surface. Such recombination at the free surface might be important in the context of cross slip because it allows the creation of the above-mentioned energetically favorable constriction alone. In addition we observe a strong preference for the partials to be in a glide plane parallel to the surface step. We have performed simulations of two screw dislocations of opposite signs, one simulation showing surface nucleated cross slip leading to subsequent annihilation of the two dislocations. It was possible to monitor the annihilation process, thereby determining the detailed dislocation reactions during annihilation. [S0163-1829(97)06630-7]
\end{abstract}

\section{INTRODUCTION}

The prominent role of dislocations and other lattice defects in controlling the mechanical properties of ductile materials is well established. Dislocation theory ${ }^{1}$ provides an understanding of the basic properties of single dislocations and, to some extent, the interaction between dislocations. However, the interactions between many dislocations can result in entangled or more ordered structures, which complicates the use of dislocation theory. In the other extreme, elasticity theory, which is the basis for dislocation theory, breaks down close to the dislocation, meaning that the interactions between two dislocations at very close range cannot be accurately described either.

One of the most famous examples of this deficiency of dislocation theory is cross slip of a screw dislocation. Cross slip is an important mechanism in plastic deformation of ductile materials. The onset of stage III in the stress-strain curves of single crystals, ${ }^{2}$ the minimum stable dipole height of screw dislocations of opposite signs, ${ }^{3}$ and the saturation of the stress in cyclic constant strain amplitude experiments 4 are examples where cross slip is believed to play a crucial role. Cross slip is a difficult problem to tackle, because it contains both long-ranged elastic interactions between dislocations and atomistic effects due to recombination of dislocations. Until now cross slip has only been theoretically investigated using methods derived from elasticity theory, thus neglecting atomistic effects.
Our approach to the problem of cross slip is nanoscale atomistic simulations involving up to 156060 atoms, thereby including both purely atomistic effects and to some extent long-range elastic interactions. Due to the length scale of the problem, cross slip has, to our knowledge, not been addressed by atomistic simulations before. Based on results from earlier nonatomistic simulations, ${ }^{5,6}$ the details of the cross-slip event are expected to be at the nanometer scale, which implies simulations involving $\sim 100000$ atoms. The use of atomistic simulations will of course dispose of the shortcomings of elasticity theory. Other problems such as finite-size effects and time-scale problems must be expected and dealt with. Furthermore, the choice of the interatomic potential is very important. Simple pair potentials all obey the Cauchy relation $C_{12}=C_{44}$ (Ref. 7) and thus cannot be expected to give a reliable description of elastic properties of specific materials. The use of a more sophisticated potential is therefore necessary. Such a potential can be derived from the effective-medium theory, ${ }^{8}$ which provides a computationally efficient interatomic potential that also includes many-atom effects.

In this paper we will be concerned with cross slip of a dissociated screw dislocation from one close-packed plane to another in an fcc metal $(\mathrm{Cu})$. We investigate bulk properties as well as the effects of having the dislocation perpendicular to a free surface. More specifically we shall focus on different possible dislocation configurations in connection with cross slip, and we determine the stress free activation energy and activation length. We show that the two constrictions 


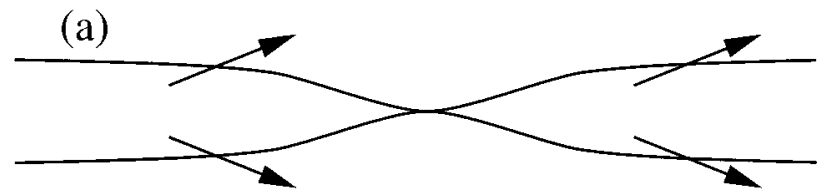

(b)

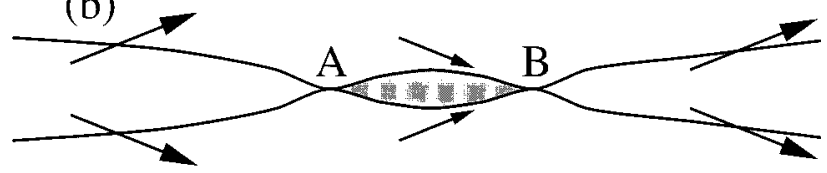

(c)

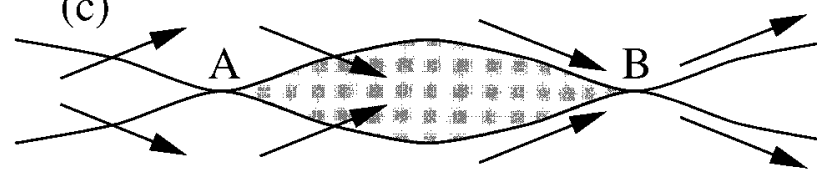

FIG. 1. The Friedel-Escaig mechanism for cross slip. (a) Creation of a Stroh-type constriction in the primary plane. (b) Dissociation in the cross-slip plane (shaded) creating two twisted constrictions $A$ and $B$. (c) Two noninteracting constrictions. The Burgers vectors are indicated with arrows. Due to the different characters of the Shockley partials, the two constrictions are not equivalent. We denote the constrictions $A$ and $B$ "edgelike" and "screwlike,", respectively.

necessary to produce cross slip in the Friedel-Escaig model $^{9,10}$ are not equivalent, and that a dislocation configuration with just one of these constrictions is energetically favored over that of two parallel Shockley partials. We also show that a free surface can act as a nucleation center for cross slip, thereby facilitating the annihilation of two screw dislocations of opposite signs. To check this we have performed simulations of two screw dislocations of opposite signs and observed their mutual annihilation. The results might serve as an atomic scale check of the approximations made in the analytical theory ${ }^{10}$ and simulations of single dislocations made within the framework of elasticity theory, 5,6 but also as input to the more mesoscopic nonatomistic simulations ${ }^{11,12}$ containing many (thousands of) dislocations, and to the modeling of experimentally characterized dislocation microstructures evolving during plastic deformation and fatigue. $^{13,14}$

The paper is organized as follows. In Sec. II we briefly present and discuss earlier work on cross slip. The methods used in this work are introduced in Sec. III. The results are presented and discussed in Sec. IV and finally we conclude in Sec. V.

\section{EARLIER WORK ON CROSS SLIP}

Several possible cross-slip mechanisms have been proposed, ${ }^{15-17}$ but recently the model by Friedel $^{9}$ and Escaig $^{10}$ (FE) has attracted most attention. In this model the dissociated screw dislocation must be recombined (constricted) over a short length, comparable to a few times the dissociation width, in the primary glide plane before the subsequent redissociation in the cross-slip plane; see Fig. 1. The FE model is derived on the basis of the line tension approximation following the method for calculating the constriction energy and shape by Stroh. ${ }^{18}$ The configuration of the partials far from the constriction, as determined in the line tension approximation, converges towards the equilibrium par-
TABLE I. Comparison between reference values of elastic constants and intrinsic stacking-fault energy and values calculated with EMT. The values for the elastic constants are in GPa. The unit for the stacking-fault energy $\gamma$ is $\mathrm{mJ} / \mathrm{m}^{2}$. The shear modulus $\mu$, calculated with EMT, is that corresponding to the $\langle 110\rangle\{111\}$ slip system.

\begin{tabular}{lccccc}
\hline \hline Element & $C_{11}$ & $C_{44}$ & $B$ & $\mu$ & $\gamma$ \\
\hline $\mathrm{Cu}$ (Ref. ) & $176.2^{\mathrm{a}}$ & $81.8^{\mathrm{a}}$ & $137^{\mathrm{a}}$ & $46,{ }^{\mathrm{b}} 59^{\mathrm{c}}$ & $70^{\mathrm{d}}$ \\
$\mathrm{Cu}$ (org. EMT) & $177^{\mathrm{a}}$ & 82.6 & 137 & 50 & 17 \\
$\mathrm{Cu}$ (mod. EMT) & 177 & 88.1 & 132 & 56 & 59 \\
\hline \hline
\end{tabular}

${ }^{\mathrm{a}}$ Ref. 38.

${ }^{b}$ Ref. 1, p. 462.

${ }^{\mathrm{c}}$ Ref. 1, p. 430.

${ }^{\mathrm{d}}$ Ref. 30.

allel separation as an exponentially decaying function. Hence, there exist a characteristic length beyond which the two constrictions $A$ and $B$ (see Fig. 1) can be considered independent and a corresponding activation energy. Escaig ${ }^{10}$ has shown that the redissociation of the screw dislocation and the motion (bow-out) in the cross-slip plane can be considered independent. It is the external stress components acting on the opposite edge characters of the Shockley partials that control the cross slip by modifying the relative splitting widths in the two glide planes. The driving force for this cross-slip mechanism is thus the widening of the stackingfault ribbon in the cross-slip plane, and no net stress on the dislocation is necessary. The process is thermally activated and the redissociation in the cross-slip plane can, in principle, take place spontaneously without any applied stress.

Experiments have been designed and carried out on copper $^{19,20}$ which, at least in a semiquantitative way, seem to confirm the FE model. However, other experiments on $\mathrm{Ge}$ and $\mathrm{Si}^{21} \mathrm{Cu},{ }^{22}$ and $\mathrm{CuAl}$ alloys ${ }^{23}$ suggest that cross slip also, maybe preferentially, nucleates at free surfaces.

One of the experimental problems is that it is impossible to actually monitor the single cross-slip event and gain detailed information about the intrinsic characteristics of the dislocation reactions. To obtain a more detailed knowledge of the structure and energetics of the cross-slipping screw dislocation, computer simulations within the framework of elasticity theory have been performed by Duesbery et al. ${ }^{5}$ and Püschl and Schoeck. ${ }^{6}$ The method of Duesbery et al. is based on numerical integration of the stresses and energies along the dislocation lines, using a relaxation method to find the equilibrium configuration of the dislocation. The method is detailed in Refs. 5 and 24. Püschl and Schoeck use the Peierls model (see, e.g., Ref. 1) to account for the atomistic structure of the dislocation core. They approximate the dislocation shape by piecewise straight dislocation segments, and by varying the geometry of the polygon-shaped dislocation they can find a minimum energy configuration.

Although their methods are different, these authors arrive at rather similar results. Both Duesbery et al. and Püschl and Schoeck find that cross slip is stress aided in the sense proposed by the FE model. However, the stress dependence seems to be overestimated in the original Escaig theory compared to these works. Using the values for the relevant parameters for $\mathrm{Cu}$ obtained in the present work (see Table I), the stress free cross-slip activation energy is found to be 2.5 
$\mathrm{eV}$ (Ref. 5) and $2.1 \mathrm{eV}$ (Ref. 6). In both cases as well as in Escaig theory the separation necessary for two constrictions to be considered independent is of the order $(50-60) b$, where $b$ is the length of the perfect Burgers vector.

The estimated activation energies are, however, subject to a considerable uncertainty for several reasons. The elastic description of a dislocation breaks down close to the dislocation line (the core). This problem is usually avoided by introducing cutoff radii in the calculations. These cutoffs are likely to be of crucial importance to the problem of cross slip which, in some way, involves recombination of the two Shockley partials. Special attention must thus be paid to the cutoff radii. In considering the influence of the cutoff radii and by using a refined version of the Stroh treatment of a constriction, Saada ${ }^{25}$ is led to conclude that the cross-slip activation energy can only be estimated to an order of magnitude within elasticity theory. Duesbery et al. also consider this problem in their work. They too find a significant dependence on the cutoff radii, both quantitatively and qualitatively.

Another uncertainty in the estimation of the activation energy from elasticity theory arises from the use of the relation for the splitting of a perfect screw dislocation into two parallel Shockley partials bounding a perfect intrinsic stacking fault,

$$
d_{0}=\frac{\mu b^{2}(2-3 \nu)}{24 \pi \gamma(1-\nu)},
$$

where $d_{0}$ is the equilibrium splitting width, $\mu$ is the shear modulus, $\gamma$ is the intrinsic stacking-fault energy, and $\nu$ is Poisson's ratio. This relation is derived on the basis of isotropic elasticity theory and can of course be expected to break down when applied in the context of an anisotropic metal such as $\mathrm{Cu}$, and it is in fact not fulfilled in our simulations. Applying relation (1) with the values for $\gamma$ and $\mu$ obtained in this work (Table I) results in an equilibrium splitting width of $d_{0} \simeq 4.5 \mathrm{~b}$. This value should be compared to the actual equilibrium splitting width obtained from the simulations of $d_{0} \simeq 6 b$ or to the experimentally determined value $^{26}$ of $d_{0} \simeq 7 b$. Using the reference values for $\gamma$ and $\mu$ also given in Table I results in $d_{0} \simeq 4 b$.

The calculated activation energies are usually given as a function of either the dimensionless variable $d_{0} / b$ or the other dimensionless variable $\gamma / \mu b$. However, the breakdown of the elasticity relation (1) means that for a particular system different estimates of the activation energy can be obtained depending on the set of variables used. To illustrate this point we can consider the results by Püschl and Schoeck. They calculate the activation energy as a function of $d_{0} / b$, and if we use the value from our simulations $d_{0} / b=6$, the above-mentioned $2.1 \mathrm{eV}$ is obtained. If we instead take the value $\gamma / \mu b=0.0042$ obtained in our simulations and use relation (1), we only get $1.4 \mathrm{eV}$. Similarly, the abovementioned activation energy of $2.5 \mathrm{eV}$ by Duesbery et al. transforms (in this case changing from $\gamma / \mu b$ to $d_{0} / b$ ) to 3.4 $\mathrm{eV}$. It is interesting to note that the analytical expression given by Escaig theory, ${ }^{20}$ when transformed into a function of either $d_{0} / b$ or $\gamma / \mu b$, results in activation energies of 1.9 $\mathrm{eV}$ or $1.3 \mathrm{eV}$, respectively. Hence, there is a systematic lowering of the activation energy when the dimensionless vari- able is changed from $d_{0} / b$ to $\gamma / \mu b$. Furthermore, there is very good agreement between the results of Escaig and those of Püschl and Schoeck, whereas the results of Duesbery et al. are roughly a factor of 2 higher.

The appeal of an approach like Escaig's lies in the fact that it provides analytical expressions for interesting properties such as activation energy and length. Even though approximate, such analytical expressions can provide physical insight that cannot be easily gained from numerical calculations. Based on the analytical theory it is possible to design specific experiments to check the theory. This has been done, ${ }^{19,20}$ and the experimental results are in fair agreement with the theory.

On the other hand, more accurate numerical calculations like the ones mentioned above may provide detailed information about the interesting properties which cannot be expected to be accurately included in the approximate theory. By suitable scaling it is even possible to achieve this information for a wide variety of materials. The numerical simulations can also serve as another check of the approximations made in the analytical theory, thereby complementing this.

The qualitative agreement between the analytical theory and the numerical simulations indicates that the essential physics of cross slip is well described within linear elasticity theory. Quantitatively the three approaches are also in fair agreement, but, as we have seen, the cross-slip activation energy is subject to rather substantial uncertainty. Even though the nonlocal part of the interactions is well described with elasticity theory, it must be emphasized that the overlap of Shockley partials and eventual recombination into a perfect screw dislocation at the constrictions is a nonelastic phenomenon, which cannot be expected to be accurately described even with the use of the most sophisticated methods derived from elasticity theory.

To avoid the uncertainties resulting from the application of elasticity theory there is no alternative to atomistic simulations. Such simulations may provide information about the partitioning of energy between elastic interactions and purely atomistic effects, and possibly reveal new phenomena which cannot be dealt with in the elastic description. Atomistic simulations can be seen as the last step in a succession of increasingly accurate but less general methods.

\section{METHODS}

\section{A. Effective-medium theory}

The effective-medium theory ${ }^{8}$ (EMT) is an approximate total energy method which has been used in describing a wide variety of phenomena at surfaces and for bulk metals including dislocation generation at high strain rates, ${ }^{27}$ deformation of nanophase metals, ${ }^{28}$ and tribology. ${ }^{29}$ In EMT the energy of an atom $i$ is calculated as the energy of the atom embedded in a suitably chosen reference system with the same electronic density as in the real system and a correction taking account of the difference between the real and reference systems.

$$
E_{i}=E_{c}\left(n_{i}\right)+E_{\mathrm{AS}}(i) .
$$

$E_{c}\left(n_{i}\right)$ is the cohesive energy of an atom embedded in an electronic density $n_{i}$ from its neighboring atoms. $E_{\mathrm{AS}}$ is the atomic-sphere correction which describes the change in en- 
ergy when the atom is moved from the real to the reference system. The atomic-sphere energy is represented as the difference between a pair potential contribution in the real and reference systems. In the present implementation of EMT the reference system is chosen to be an fcc crystal. The parameters entering into the EMT potential can be found from either first-principles calculations or from fitting to experimental values of bulk elastic and cohesive properties. ${ }^{8}$ Generally EMT describes the elastic properties of metals quite well, but for $\mathrm{Cu}$ the energy of an intrinsic stacking fault $\gamma$ is much underestimated. In the context of dislocation reactions this low value of the intrinsic stacking-fault energy might be a serious drawback. The energy contributions from different atomic layers to the total stacking-fault energy have been calculated $^{30}$ using density functional theory for a range of transition and noble metals. An analysis ${ }^{31}$ of these calculations shows that the energetics can be understood in a simple two-parameter model. The two parameters describe the relative contribution to the stacking-fault energy from the $s$ and $d$ electrons and the results of the model are in excellent agreement with first-principles calculations. ${ }^{30}$ The parameter describing the contribution from the $s$ electrons can be approximated by a pair potential. Furthermore, it is shown that for the noble metals $(\mathrm{Au}, \mathrm{Ag}$, and $\mathrm{Cu}$ ) this parameter is the main contributor to the stacking-fault energy. With this knowledge, it was decided to modify the original EMT atomic-sphere potential by a small additional pair potential fitted so as to reproduce a reasonable intrinsic stacking-fault energy. The potential was chosen to be Gaussian shaped and located around the fifth-nearest neighbor in a perfect hcp stacking sequence. This corresponds to a distance of $\sqrt{(11 / 3)} d_{\mathrm{NN}}$ where $d_{\mathrm{NN}}$ is the nearest-neighbor distance in an fcc crystal. As the modification is small in amplitude it was decided to keep all the original EMT parameters unchanged. In Table I we show values of relevant elastic constants for $\mathrm{Cu}$ calculated with both the original and modified EMT potentials. For comparison we also quote experimental values and the value for the intrinsic stacking-fault energy obtained by the above mentioned first-principles calculation. The calculated $\mu$ is the shear modulus corresponding to the $\langle 110\rangle\{111\}$ slip system. The overall quality of the EMT potential is unaffected by the modification, and the calculated values with the modified EMT potential are all within $\sim 15 \%$ of the reference values.

\section{B. Geometry of the system}

In all simulations the overall geometry of the systems is basically the same, and is shown in Fig. 2. Only the dimensions of the systems and whether the initial screw dislocation is perfect or dissociated in one or two of the two possible glide planes vary. The systems contain a centered screw dislocation with $b=\frac{1}{2}[110]$, and may thus be seen as a stacking of (110) planes. The screw dislocation is introduced in the otherwise perfect fcc crystal by using the result for the displacement of the atoms from linear elasticity theory,

$$
u_{b}=\frac{b \theta}{2 \pi},
$$

where $\theta$ is the angle between a fixed direction and the vector from the dislocation to the actual atom. Since the fcc stack-

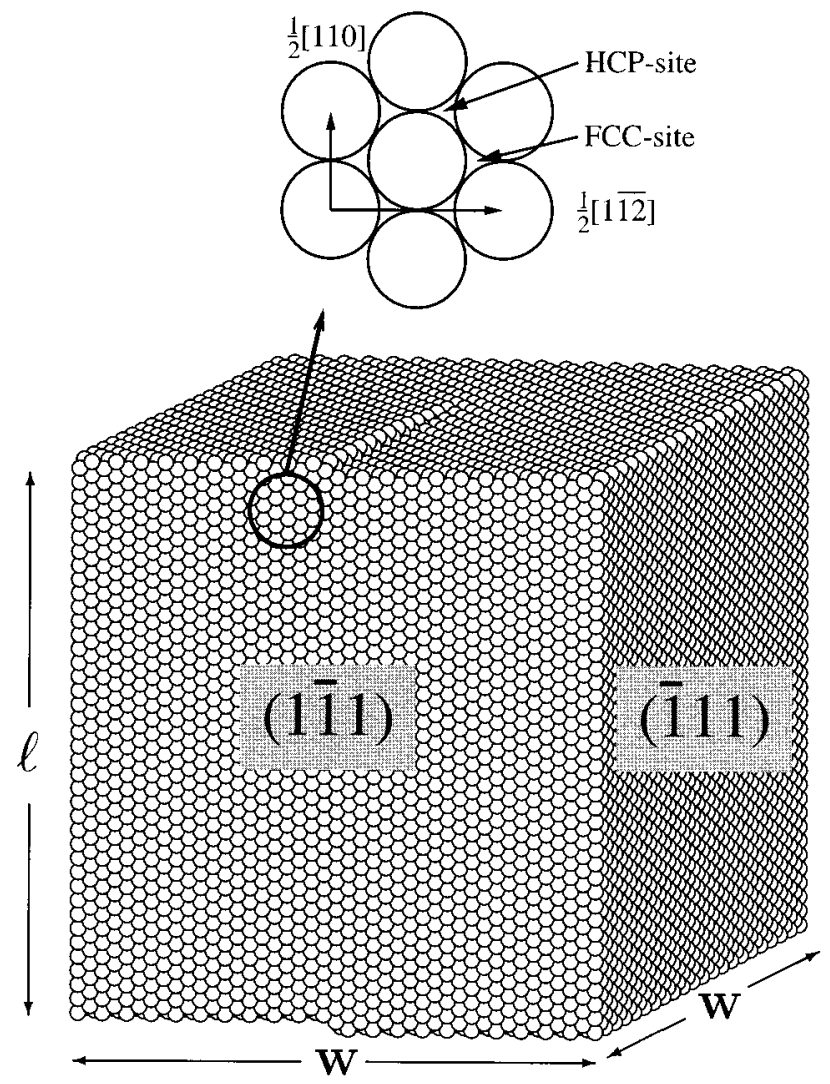

FIG. 2. A medium-size system consisting of 73960 atoms. The system contains one centered screw dislocation parallel to the [110] direction perpendicular to the top surface. The surface step is parallel to the $(\overline{1} 11)$ plane. The width of the two $\{111\}$ planes is $w=9.5 \mathrm{~nm}$, and the length of the dislocation (the height of the crystal) is $\ell=40 b(10.2 \mathrm{~nm})$. The inset shows the difference between fcc and hcp sites in the (1 11$)$ plane.

ing sequence in the [110] direction is $\ldots A B A B \ldots$, this results in two intertwined helix-shaped [110] planes. The two Shockley partials are introduced as screw dislocations with Burgers vectors equal to half the Burgers vector of the perfect screw dislocation, and they can be in either glide plane with variable initial separation. It is also possible to prepare the system with partials of varying separation, e.g., like any of the shapes shown in Fig. 1. In this latter case, the partials are approximated with straight dislocation segments before calculating the displacements. The splittings in the two glide planes are as follows:

$$
\begin{aligned}
& (1 \overline{1} 1) \text { plane: } \\
& \frac{1}{2}[110] \rightarrow \frac{1}{6}[121]+\frac{1}{6}[21 \overline{1}], \\
& (\overline{1} 11) \text { plane: } \quad \frac{1}{2}[110] \rightarrow \frac{1}{6}[211]+\frac{1}{6}[12 \overline{1}] .
\end{aligned}
$$

In Fig. 2 we show a system consisting of 73960 atoms. The system is made up by a stacking of 80 (110) planes, but might as well be regarded as a stacking of 43 (111) planes or 43 (111) planes. The length of the dislocation (the height of the system) is denoted $\ell$, and the width of the two $\{111\}$ planes $w$. The two different $\{111\}$ planes are not perpendicular; rather, the angle between them is $70.53^{\circ}$. The $\{111\}$ sur- 

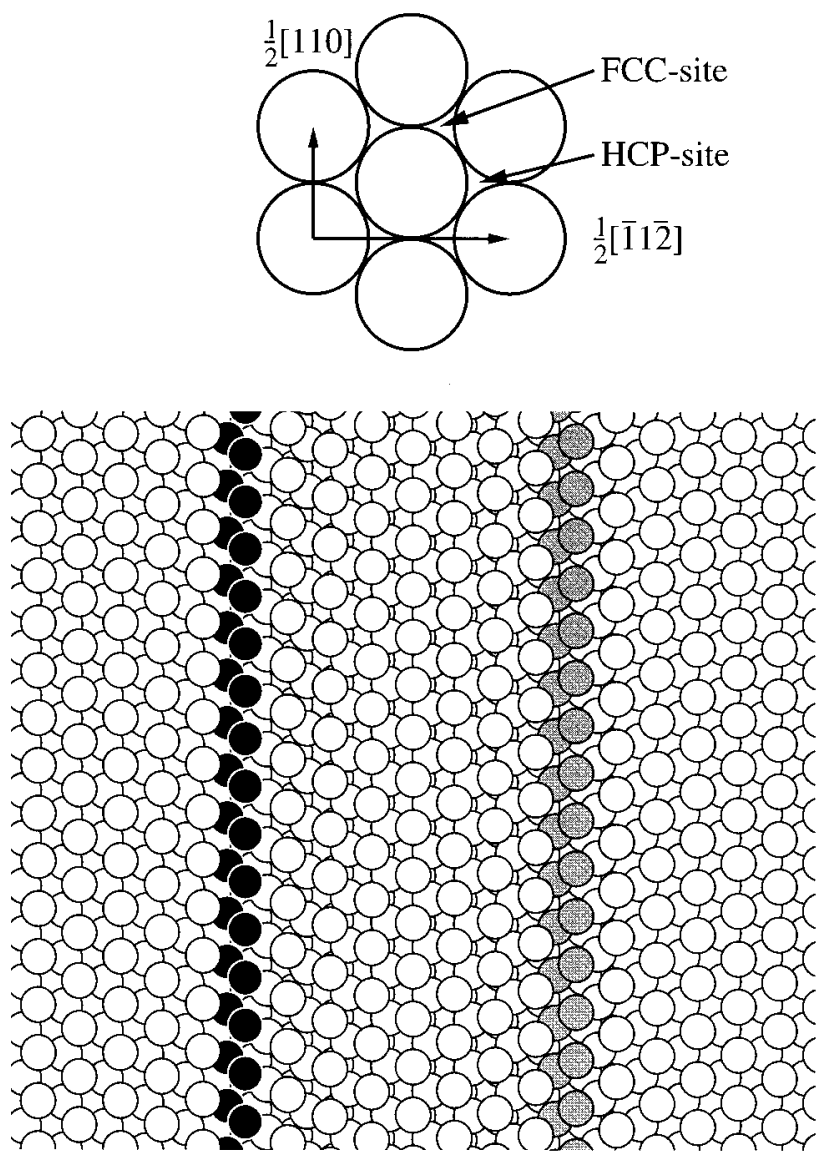

FIG. 3. Zoom-in on a (111) plane with the two Shockley partials colored black and grey. The system has been sliced through its middle, and the radius of the atoms has been reduced to enable inspection of the stacking sequence. Perfect fcc stacking is seen to the left and to the right of the partials. The stacking changes gradually from fcc to bridge (at the partials) to hcp between the partials. The inset shows the difference between fcc and hcp sites in the (111) plane.

faces are always free. The steps on the two (110) surfaces are parallel to a (111) plane, and the dislocation is located in the middle of the system. In Sec. IV A we always apply periodic boundary conditions along the [110] direction and the position of the step is thus irrelevant. However, in Sec. IV B we remove the periodic boundary conditions and the orientation of the step becomes important. By bulk simulations (Sec. IV A) we mean simulations with periodic boundary conditions along the [110] direction, even though the $\{111\}$ surfaces are free. We will refer to simulations without any periodic boundary conditions as free surface simulations (Sec. IV B), meaning that one or two of the (110) surfaces are now free.

The two $\{111\}$ planes are not exactly equivalent with respect to a given direction, e.g., the [110] direction, and this results in two different configurations of the Shockley partials in the two possible glide planes. This difference is a result of the two kinds of hollow sites, i.e., the fcc site and the hcp site, in a $\{111\}$ plane. For the (111) plane and the (111) plane the fcc sites are geometrically different with respect to, e.g., the [110] direction. In Fig. 3 we show a close-up of a (111) plane with two Shockley partials, and it
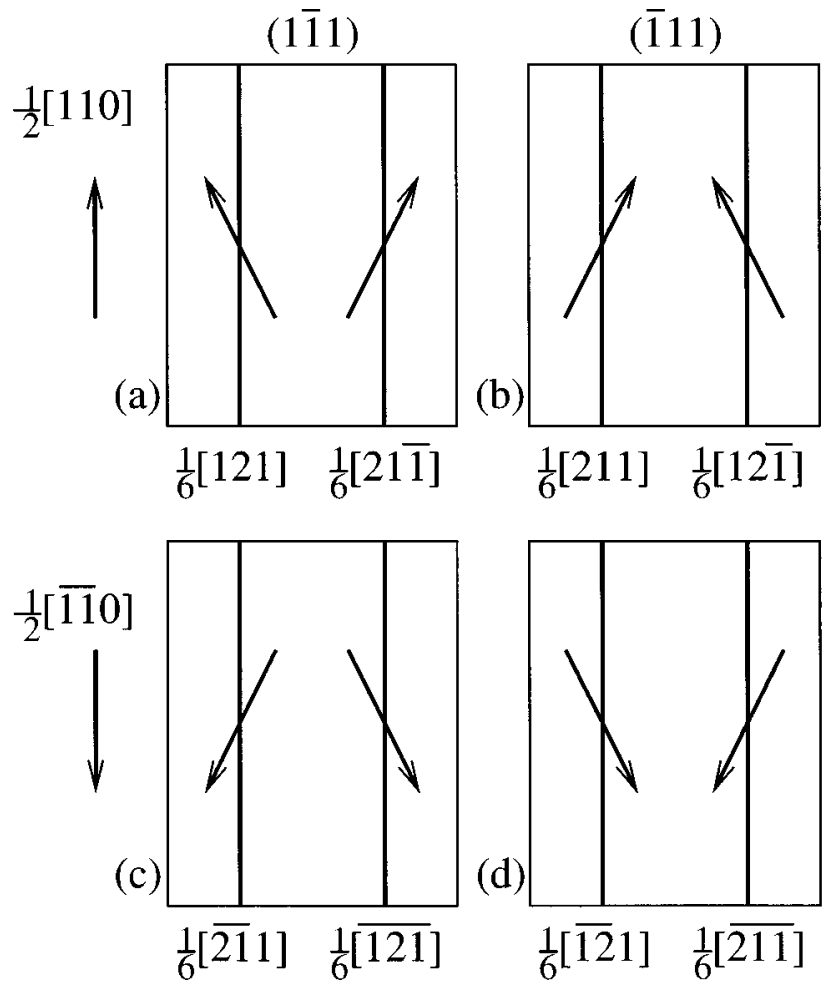

FIG. 4. Configuration of the Shockley partials in the two possible glide planes: (a) (1 $\overline{1} 1)$ plane, $b=\frac{1}{2}[110]$. (b) (1ㅣ) plane,

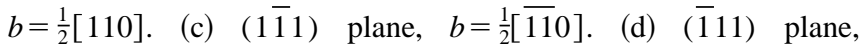
$b=\frac{1}{2}[\overline{11} 0]$.

is possible to inspect the detailed stacking sequence. The perfect fcc stacking is to the left and to the right of the partials and the perfect Burgers vector $\frac{1}{2}[110]$ points upwards in the figure. The atoms in fcc sites closest to the viewer are located in hollow sites surrounded by three atoms in the underlying plane comprising a triangle pointing to the right in the figure, whereas for the hcp sites between the partials the corresponding triangle points to the left. For the (1iㅣ) plane the fcc site is different and the underlying triangle points to the left; see Fig. 2. When the perfect screw dislocation dissociates, this results in two different configurations of the Shockley partials. In the (1 $\overline{1} 1)$ plane the partial Burgers vectors point away from each other, whereas in the (111) plane the partial Burgers vectors point toward each other. This can easily be understood in the following manner. In the $(1 \overline{1} 1)$ plane the sequence of the partials is dictated by the formation of the stacking fault in between them. The symmetry operation that transforms the (1 $\overline{1} 1)$ plane into the (111) plane is just a rotation around the [110] direction followed by a $180^{\circ}$ rotation around the plane normal. The latter rotation reverses the dislocation orientation, which means that the signs of the rotated Burgers vectors must be reversed too. In Fig. 4 we show the splittings of the perfect screw dislocation into Shockley partials in the two different glide planes.

\section{Identification of dislocations}

In a study like this it is of course essential that we can identify the dislocations. For this purpose we use a disloca- 
tion finding program developed by Schiøtz. ${ }^{32}$ The basic idea is to treat the dislocation as a topological defect as in dislocation theory. For each atom small "Burgers circuits" are made in the vicinity of the atom, thereby identifying the atoms close to the dislocation line. These atoms can then be colored by a visualization tool. Atoms near the surface of the system are also given a "Burgers-vector" by the program, which causes some of these atoms to be colored together with the actual dislocation atoms. For details about the method we refer to Ref. 32. The program has been used in simulations involving different defects, e.g., migrating grain boundaries ${ }^{28}$ and high velocity dislocations. ${ }^{27}$

\section{Simulation methods}

The simulations are either molecular-dynamics (MD) simulations at a finite temperature or energy-minimization simulations at zero temperature. The simulations in Sec. IV B 3 were performed using the Langevin algorithm, where a small fluctuating force and a friction term stabilize the temperature at $580 \mathrm{~K}$. All other finite-temperature simulations were performed at room temperature (RT) using the Verlet algorithm after the temperature had been stabilized by an initial application of Andersen thermalization. Finally we also use direct minimization of the energy by a moleculardynamics (MD) minimization algorithm (MDmin). The MDmin algorithm performs a Verlet MD time step and zeros the momentum whenever the dot product between the momentum and the force is $<0$, thereby bringing the system to an energy minimum. The length of an MD time step is $5.4 \times 10^{-15} \mathrm{~s}$. For details about the MD algorithms and the employed MD simulation tool and visualization tool, we refer to Ref. 33.

\section{RESULTS AND DISCUSSION}

\section{A. Bulk simulations}

\section{Splitting width}

As a starting point the equilibrium splitting width $d_{0}$ of the dissociated screw dislocation was determined. This will serve as yet another check of the reliability of the interatomic potential. $d_{0}$ was found by varying the width $w$ and the length $\ell$ of the computational cell, using both direct minimization of the energy and RT simulations. Periodic boundary conditions were applied in the [110] direction (along the dislocation line) and a perfect screw dislocation as well as two Shockley partials of variable initial splitting width in both $\{111\}$ planes were used as initial configurations. In all cases $d_{0}=7 \pm 1 k$, where $k$ is the length of a $\frac{1}{4}\langle 112\rangle$ vector. At room temperature this corresponds to $d_{0}=1.5 \pm 0.2 \mathrm{~nm}$, in very good agreement with the experimental result ${ }^{26}$ of $d_{0}=1.8 \pm 0.6 \mathrm{~nm}$. Using the elastic constants from Table I and the isotropic elasticity relation (1) to determine the equilibrium splitting width results in $d_{0}=1.1 \pm 0.1 \mathrm{~nm}$.

A prominent feature of the splitting of the perfect screw dislocation is the in-plane smearing of the Shockley partials which strongly influences the stacking between them. This smearing is also observed in computer simulations ${ }^{34-36}$ of edge dislocations in $\mathrm{Cu}$. In Fig. 3 the radius of the atoms has been reduced to allow inspection of the stacking sequence, and it is easily seen how the partials invade the ribbon be- tween them. The stacking changes from fcc sites to the left and to the right of the partials to bridge sites at the partials and a few columns of hcp sites between the partials. In principle it could be possible to fit the displacements to a Peierls model of the dislocation core, and obtain a value for the dislocation "width." The width of a single Shockley partial can also be roughly estimated from Fig. 3 to be $\sim 4 k \simeq 0.9$ $\mathrm{nm}$. The effect of the in-plane smearing is to prevent the faulted ribbon between the partials from being a perfect intrinsic stacking fault, but also to increase the total width of the fault compared to the separation of the partials. However, the in-plane smearing is only $\sim 60 \%$ of the total splitting width, indicating that the partials are well separated. If anisotropy can be neglected, the application of relation (1) should be valid. The equivalent relation ${ }^{1}$ for the splitting of a perfect edge dislocation into two Shockley partials predicts that separation to be $\sim 2.5 \mathrm{~nm}$, which should be compared to the experimental result ${ }^{26}$ of $3.8 \pm 0.6 \mathrm{~nm}$. Using anisotropic elasticity theory ${ }^{37}$ with a stacking-fault energy of $70 \mathrm{~mJ} / \mathrm{m}^{2}$ results in splitting widths for a screw and an edge dislocation of $1.3 \mathrm{~nm}$ and $4.1 \mathrm{~nm}$, respectively, in better agreement with experiment and our result for the screw dislocation. Hence, the incapability of relation (1) of reproducing the experimentally found splitting widths must be attributed to the fact that this relation is derived from isotropic elasticity theory.

\section{Cross-slip activation energy}

The models based on elasticity theory estimate the crossslip activation energy to approximately $2 \mathrm{eV}$. This energy is much too high to produce a rate of cross slip which can be simulated with ordinary MD simulations, where the typical time scale of a simulation is $10-100$ ps. However, it is possible to simulate different optimized static configurations of the dislocation, and thereby obtain information about the structures and energies. To determine the stress-free crossslip activation energy in the FE model we divide the problem in two. The reason for this lies again in the length scale of the problem. The separation between the two constrictions in the transition state is expected to be $55 b(14.0 \mathrm{~nm})$ which implies the use of a computational cell at least twice this length. We therefore decided to make simulations of isolated constrictions in computational cells of varying sizes. As can be seen in Fig. 1, the two constrictions necessary in the FE model are not equivalent. At constriction $B$ the partials assume screwlike character, whereas at constriction $A$ the partials are more like edge dislocations. We shall distinguish between these two constrictions and refer to them as "screwlike" and "edgelike," respectively.

We shall define the energy of a constriction as the difference in minimized energy of the constricted dislocation and the minimized energy of two parallel Shockley partials of the same length, $\ell$. The procedure of determining the constriction energy then consists of two steps: First, we minimize the energy of two reference systems, each containing a pair of parallel Shockley partials in the $(1 \overline{1} 1)$ plane and the $(\overline{1} 11)$ plane, respectively. Second, we extract the four top and four bottom (110) planes of the relaxed configurations. Taking the top planes and bottom planes from different relaxed reference systems and making a sandwich with a system containing an initially constricted (and twisted) dislocation gives us one constriction. Taking the other top and bottom set with 


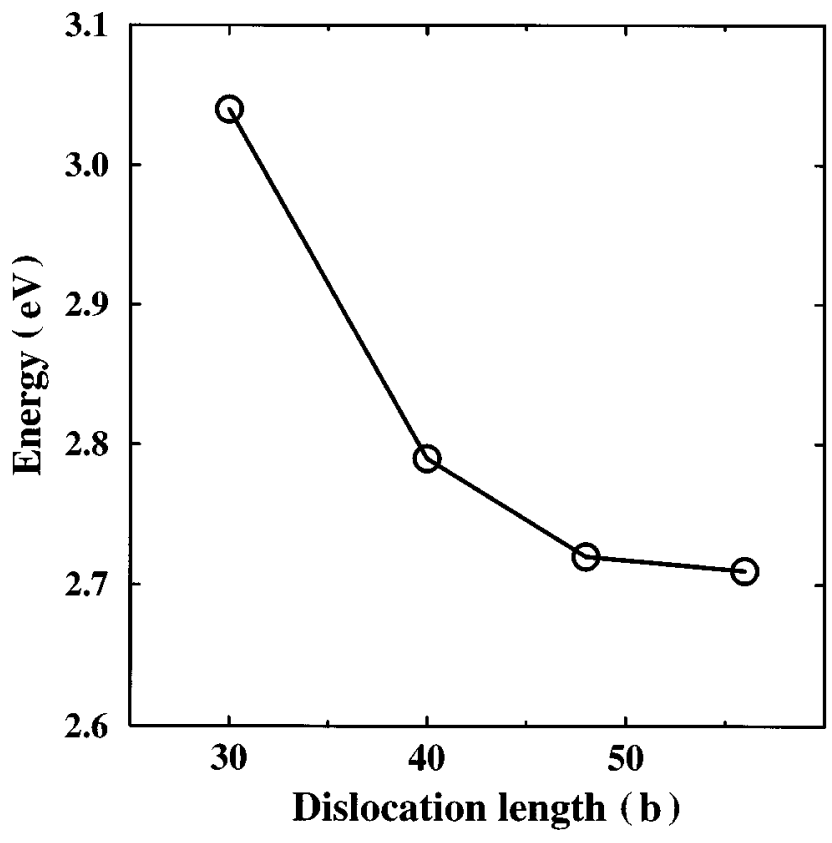

FIG. 5. Total energy in $\mathrm{eV}$ of the two constrictions as a function of dislocation length $\ell$. The width of the systems is kept fixed, $w=11.3 \mathrm{~nm}$. The energy converges for $\ell \sim 50 b$.

another initially constricted system gives us the second constriction. It is important to keep the four top planes and the four bottom planes static; i.e., the atoms are not allowed to move during the simulation. By using the top and bottom from the already relaxed systems as static atoms, we are able to mimic a semi-infinite bulklike dislocation on either side of the constricted part. The choice of four static planes ensures that the dynamic atoms do not feel the vacuum above and below the static layers. We have performed simulations with dislocation lengths in the range (30-60) $b(7.7-15.3 \mathrm{~nm})$ and with the width perpendicular to the dislocation in the range $7.7-14.8 \mathrm{~nm}$. The smallest system consisted of 36750 atoms and the largest system consisted of 156060 atoms. Calculating the two constriction energies for each system size then requires energy minimization of 4 systems, including the relaxation of the two reference systems.

It is obvious that the constraint of equilibrium separation distance between the partials imposed by the static layers makes the energy of the constrictions depend on the length of the computational cell, $\ell$. This dependence is, however, expected to vanish as $\ell$ is increased, thereby making the constriction energies converge. To check this we performed simulations with a fixed width of $w=11.3 \mathrm{~nm}$ and $\ell$ varying from $30 b$ to $60 b$. The results of these simulations are shown in Fig. 5. The convergence of the energy is clearly seen, and we can estimate a minimum length, i.e., a minimum distance between noninteracting constrictions, of $\sim 50 b$. To check the dependence of the energies on the width of the system, we performed simulations with a fixed length $\ell=30 b$ and widths in the range 7.7-14.8 $\mathrm{nm}$. These simulations showed no dependence of the energies on the widths for widths larger than $10 \mathrm{~nm}$. For systems with widths less than $10 \mathrm{~nm}$, we observed a small increase in the energies. Based on these observations we can conclude that the minimum separation between independent constrictions is $\sim 50 \mathrm{~b}$, and that the cross-slip activation energy is $2.7 \mathrm{eV}$. This result for the activation energy agrees well with the result of Duesbery et al. ${ }^{5}$ mentioned in Sec. II, and it is somewhat higher than the estimates of Escaig ${ }^{20}$ and Püschl and Schoeck. ${ }^{6}$ Also, the minimum separation between noninteracting constrictions is in perfect agreement with all three estimates based on elasticity theory. For clarity we only show the sum of the constriction energies in Fig. 5. Taken separately the constriction energies converge similarly to the sum, but they show a very interesting difference: The energy of the edgelike constriction is $3.8 \mathrm{eV}$, whereas the energy of the screwlike constriction is negative, $-1.1 \mathrm{eV}$. None of the mentioned works based on elasticity theory treat the constrictions separately, making a detailed comparison impossible.

The most striking result is the negative energy of the screwlike constriction. This indicates that the configuration with the dislocation in the constricted and twisted screwlike state is energetically more favorable than just two parallel Shockley partials in one glide plane. Intuitively it is obvious that the screwlike constriction must be energetically preferred over the edgelike constriction. In dislocation theory it is well established that the self-energy of a screw dislocation is lower than that of an edge dislocation. It is also known ${ }^{1}$ that the interaction energy between two perfect screw dislocations inclined at an angle of $45^{\circ}$ is zero. The angles between the partials involved here are not exactly $45^{\circ}$, but they are not very far from that. Finally the constriction reduces the area of the stacking fault, thereby lowering the energy for both constrictions.

The atomistic approach enables an even more detailed investigation of the energetics than that described above. It is possible to plot the energy of the systems layer by layer, thereby gaining insight into the "energy distribution" along the dislocation line. The decomposition of the total energy into contributions from individual atoms is of course, in principle, not unique. However, within the EMT there is a natural way of doing this. ${ }^{8}$ The energy of an $A B$ (110) plane pair is defined as the difference in energy between an $A B$ pair in the relaxed reference systems containing parallel partials and the actual $A B$ pair in the constriction system. The $A B$ pairs in the reference systems all have the same energy, whereas the energy of the $A B$ pairs in the constricted systems are expected to vary along the dislocation line. In Fig. 6 we show the results of such a projection of the energy onto the individual $A B$ pairs for the systems consisting of 156060 atoms having $w=11.3 \mathrm{~nm}$ and $\ell=60 b(15.3 \mathrm{~nm})$. We only show the energy of the dynamic atoms. In this type of plot the qualitative difference between the constrictions is very apparent. Returning to the intuitive considerations about constriction energies given above, we can try to explain the behavior. For the screwlike constriction there is a drop in energy towards the center of the system. This might be explained by the lowering of the self-energy of the partials as they assume a more screwlike orientation, the reduction in interaction energy also due to acquired screw orientation, and, finally, the reduction in stacking-fault area. For the edgelike constriction only the reduction in stacking-fault area would tend to reduce the energy. The acquirement of a more edgelike orientation would, according to dislocation theory, cause the energy to increase. Common to the two constrictions is, however, a peak in energy located around the center of the system. A probable explanation is that atomistic 


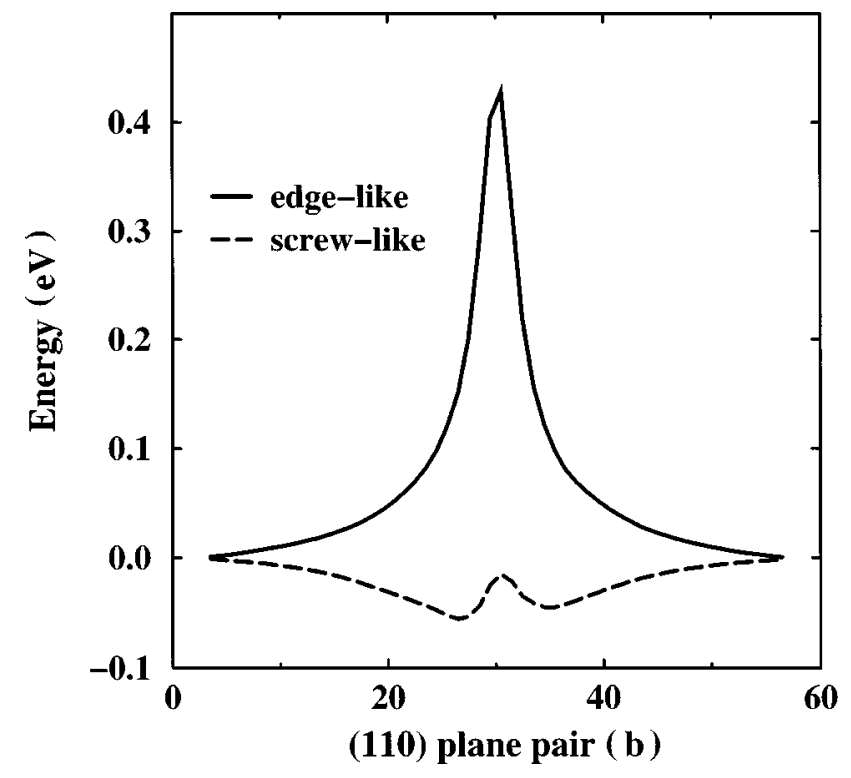

FIG. 6. Energy contribution from individual (110) plane pairs to the total energy of the two kinds of constrictions. Upper curve: edgelike constriction. Lower curve: screwlike constriction. The length of the dislocations is $\ell=60 b(15.3 \mathrm{~nm})$ and the width of the systems is $w=11.3 \mathrm{~nm}$. The systems consist of 156060 atoms. Only the contributions from the dynamical atoms are shown. Notice the negative energy of the screwlike constriction.

effects begin to play a role. Such effects could be caused by the increasing overlap of the cores of the partials and the eventual recombination into a perfect screw dislocation at the center of the system. The extension along the dislocation line of this "atomistic domain" can be estimated from the figure to be approximately $6 b$. This rough estimate fits nicely with the pictures (see Fig. 7) of the constrictions, where the extension of the recombined domain is also about $(5-6) b$ by visual inspection.

In Fig. 7 we show relaxed configurations of both constriction types. The systems are the same as those in Fig. 6. The two constriction types cannot be distinguished visually. From pictures similar to Fig. 7 it is possible to estimate the length over which the partials constrict simply by counting the number of planes where the partials are nonparallel. The counting was done using pictures with reduced atomic radii and no coloring of the atoms, i.e., not using Fig. 7. For both constrictions the result is $(25-30) b(6.4-7.7 \mathrm{~nm})$. Decreasing the length $\ell$ of the dislocation to $40 \mathrm{~b}$ does not change the visual nonparallel lengths significantly. These nonparallel lengths are representative for all the systems we have used. A nonparallel length of $\sim 30 b$ indicates that this is the minimum reasonable length of the dislocations in simulations of this kind.

The negative energy of the screwlike constriction raises some interesting questions about the behavior of a screw dislocation: For example, will screw segments in a dislocation network dissociate into Shockley partials in two glide planes connected by a screwlike constriction? And how is the mobility of a screw dislocation affected by the presence of a screwlike constriction? Another interesting aspect is the apparent lack of experimental evidence of the existence of
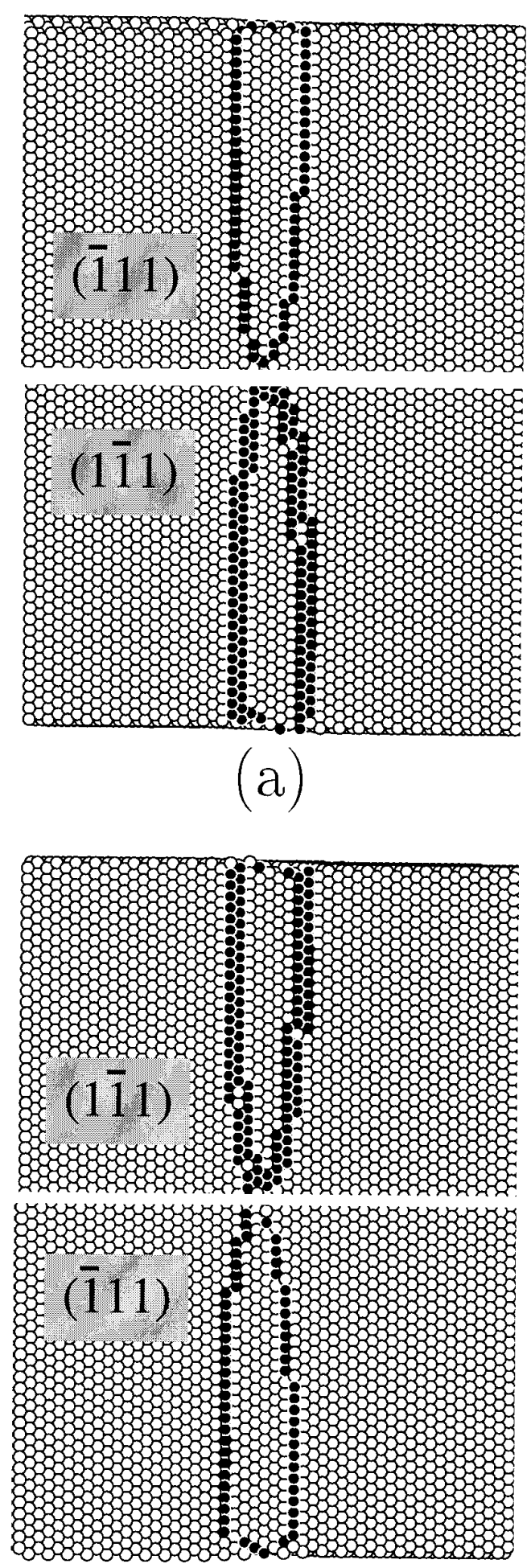

(b)

FIG. 7. Relaxed configurations of both constriction types. The systems are the same as those in Fig. 6. The systems have been cut in halves and rotated to display the dislocation in both glide planes.

screwlike constrictions. In Sec. IV B we show how cross slip can be initiated by the creation of a screwlike constriction near a free surface. A direct consequence of such surface nucleated cross slip is the annihilation of two screw dislocations of opposite signs. 

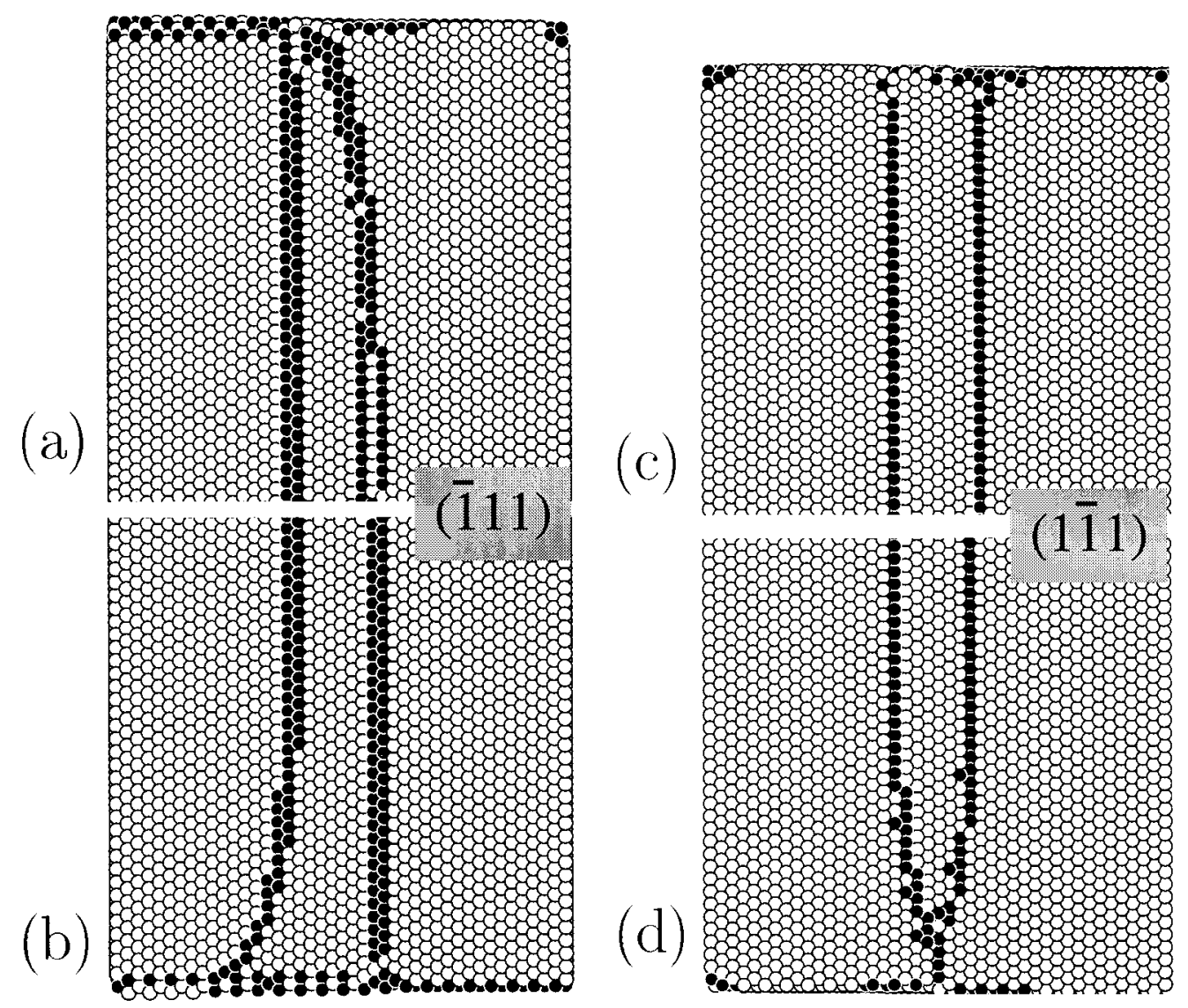

FIG. 8. Four different systems with one free (110) surface after direct energy minimization: (a) (111) plane, static atoms in the bottom of the crystal. (b) ( $\overline{1} 11)$ plane, static atoms in the top of the crystal. (c) (111) plane, static atoms in the bottom of the crystal. (d) (1 $\overline{1} 1)$ plane, static atoms in the top of the crystal. The dislocation has cross slipped in the bottom of the crystal, thereby creating a screwlike constriction. The differences between the two glide planes are due to the different orientation of the surface step relative to the glide planes.

\section{B. Free surface simulations}

\section{One dislocation: One free surface}

Experiments ${ }^{21-23}$ have shown that cross slip of screw dislocations also, maybe preferentially, takes place at free surfaces. The effect of a free surface on the configuration of a dissociated dislocation is very different from usual bulk behavior. To investigate the role of a free surface, Hazzledine and co-workers ${ }^{23}$ performed transmission electron microscopy (TEM) experiments on low-stacking-fault-energy $\mathrm{CuAl}$ alloys and used elasticity theory to address the problem theoretically. According to the theory the free surface acts in such a way as to modify the interactions between the partials in two ways. In the vicinity of a free surface the partials attract and they tend to rotate towards screw character. For a dissociated screw dislocation running through a slab and emerging at free surfaces on both sides of the slab, these two effects will compete on one side of the slab and reinforce on the other. The experimental results showed good agreement with the predictions of the theory with a preference of the partials to acquire screw character. In order to investigate the role of a free surface qualitatively, we have made simulations where the dislocation is perpendicular to one or two free (110) surfaces.

The simulations with just one free surface were performed in the same fashion as the simulations containing a constric- tion. By removing the periodic boundary conditions from the already relaxed configuration containing two parallel partials and making, e.g., the four top (110) layers static, it is possible to mimic a semi-infinite rod with one dissociated screw dislocation ending at a free (110) surface in the bottom of the crystal. Having the dislocation initially in either of the two possible glide planes gives four different systems with one free (110) surface. The two systems with two free (110) surfaces were generated simply by removing the periodic boundary conditions from the already relaxed reference systems, thereby creating clusters with a single dislocation emerging on both free (110) surfaces. All the systems had $w=9.5 \mathrm{~nm}$ and $\ell=40 b(10.2 \mathrm{~nm})$ and consisted of 73960 atoms. We performed direct energy minimization as well as RT simulations followed by a quench by MDmin energy minimization.

Figure 8 shows the four systems with one free (110) surface after the direct energy minimization. For the two systems with the partials initially parallel in the (111) plane and with static atoms in the bottom of the crystal [Fig. 8(a)] or in the top of the crystal [Fig. 8(b)], the predicted effects of the free surface are clearly seen. In the (111) plane the partial Burgers vectors point toward each other as seen in Fig. 4(b). For the system with static atoms in the top, one of the Shockley partials bows out away from the other thereby acquiring 
more screw character, whereas the other partial stays straight. The tendency to acquire more screw character seems to be stronger than the tendency for the partials to attract, in agreement with the experimental findings. ${ }^{23}$ However, it is not easy to judge which of the effects is the stronger because of the interaction with the image dislocations parallel to the real dislocation, which would also tend to attract the partials to the free $(1 \overline{1} 1)$ surfaces. With the static atoms in the bottom of the crystal, the effect of the free surface is to make the two partials recombine into a perfect screw dislocation in the top of the crystal. There is no sign of redissociation of the perfect screw dislocation in the (111) plane. The recombination in the $(\overline{1} 11)$ plane is a result of one or both of the effects of a free surface predicted by elasticity theory, and together these two effects are stronger than the interaction with the parallel image dislocations. Again one of the partials bows out to acquire more screw character, whereas the other partial stays straight.

The asymmetrical configurations of the partials near the free surfaces in Figs. 8(a) and 8(b) must be attributed to the asymmetrical positions of the partials with respect to the surface step. Furthermore, the two $\{111\}$ glide planes are not equivalent with respect to the step on the free (110) surfaces. The surface step is located in a (111) glide plane; see Fig. 2. When the partials are in this plane the step is parallel to the partials. However, when the partials are in a (1.11) glide plane the surface step is located between the partials at an angle of $70.53^{\circ}$.

For the two systems with the Shockley partials initially parallel in the (111) plane, the effect of the free surface is therefore somewhat different. In this plane the partial Burgers vectors point away from each other; see Fig. 4(a). The system with static atoms in the top of the crystal [Fig. 8(d)] corresponds to Fig. 8(a). The two partials recombine as expected into a perfect screw dislocation in the bottom of the crystal, but in this case we observe an additional splitting of the perfect screw dislocation in the $(\overline{1} 11)$ plane. The redissociation in the (111) plane, not visible in Fig. 8(d), generates a screwlike constriction on the dislocation and allows the partials to be in a glide plane parallel to the surface step. Apparently there is no or a very-low-energy barrier for this kind of surface nucleated cross slip. For the system with static atoms in the bottom [Fig. 8(c)] no net effect of the free surface is observed. The partials stay straight, even very close to the free (110) surface. The tendency to rotate towards screw character seen in Fig. 8(b) seems to be compensated by an attraction to the surface step.

For all four systems we have also performed RT simulations followed by a quench by MDmin minimization. These simulations show no qualitative differences from the above mentioned simulations. The only difference is for the system with static atoms in the top of the crystal and the partials initially in the (1) $\overline{1} 1)$ plane which shows surface nucleated cross slip. The redissociation in the $(\overline{1} 11)$ plane in the bottom of the crystal is much more pronounced, with the partials in the lower part of the crystal in the $(\overline{1} 11)$ plane adopting a configuration almost identical to that of Fig. 8(a).

\section{One dislocation: Two free surfaces}

In order to obtain more information about the influence of free surfaces on the configuration of the partials, we also performed RT simulations followed by a quench by MDmin minimization on systems with two free (110) surfaces; i.e., the systems were clusters with the partials initially in either the (111) plane or the (1 $\overline{1} 1)$ plane. From the simulations of systems with one free (110) surface, we expect the first system to recombine into a perfect screw dislocation in the top of the crystal and to split even further than the bulk splitting in the bottom of the crystal. For the latter system we expect the system to perform cross slip in the bottom of the crystal, but it is not clear whether the cross slip will be complete or whether the system prefers a configuration with a screwlike constriction. The system with the partials initially in the (111) plane behaves as expected. The partials recombine in the top of the crystal with no sign of redissociation in the (1) 11$)$ plane and they repel in the bottom of the crystal. After $\sim 11$ ps (and before the quench) the dislocation is located off center in the crystal, due to the attraction to the image dislocation; see Fig. 9(a). The system with partials initially in the (1) 11$)$ plane performs a complete cross slip to the $(\overline{1} 11)$ plane. The partials recombine and redissociate in the bottom of the crystal, thereby creating a screwlike constriction. The screwlike constriction ascends the crystal, making the partials in the top gradually change their glide plane from the (1) 11$)$ plane to the $(\overline{1} 11)$ plane. The cross slip is completed in $\sim 11$ ps. Figure 9(b) shows an intermediate stage in the cross slip and Fig. 9(c) shows the final relaxed configuration of the cross-slipped dislocation after the quench. Figures 9(a) and 9 (c) are very similar to TEM pictures of dissociated screw dislocations in thin foils in Ref. 23.

To check the significance of the orientation of the surface step with respect to the partials we performed simulations similar to the two simulations with two free surfaces, but this time with the step parallel to a (1 $\overline{1} 1)$ plane; i.e., the surface step has been rotated compared to the step seen in Fig. 2. The results are equivalent to the simulations with the original step orientation. Now the system with the partials initially in the (111) plane cross slips to the (111) plane, while the system with the partials initially in the (111) plane, which is now parallel to the step, does not. The two final configurations have the partials recombined in the bottom of the crystal and split in the top of the crystal.

These simulations show that there is a strong preference for the dissociated screw dislocation to be in the glide plane parallel to the surface step, and that the dislocation will adopt a configuration with perfect screw dislocation in one end and two nonparallel Shockley partials in the other end. It is the desire of the partials to be in a plane parallel to the surface step and not the possibility of making a screwlike constriction, which controls the surface-nucleated cross slip, and there will be an energy barrier for surface nucleated cross slip away from the glide plane parallel to the surface step.

Finally we performed simulations on systems with different step orientations in either end of the crystal. With the partials initially in the $(\overline{111})$ plane or the $(1 \overline{1})$ plane this gives four possibilities. For the two systems with the step parallel to the (1) $\overline{1}$ ) plane in the top and the step parallel to the (111) plane in the bottom we expect the dislocation to adopt a configuration with a screwlike constriction. This configuration will allow the partials to be parallel to the step in both ends of the crystal. However, a similar configuration 

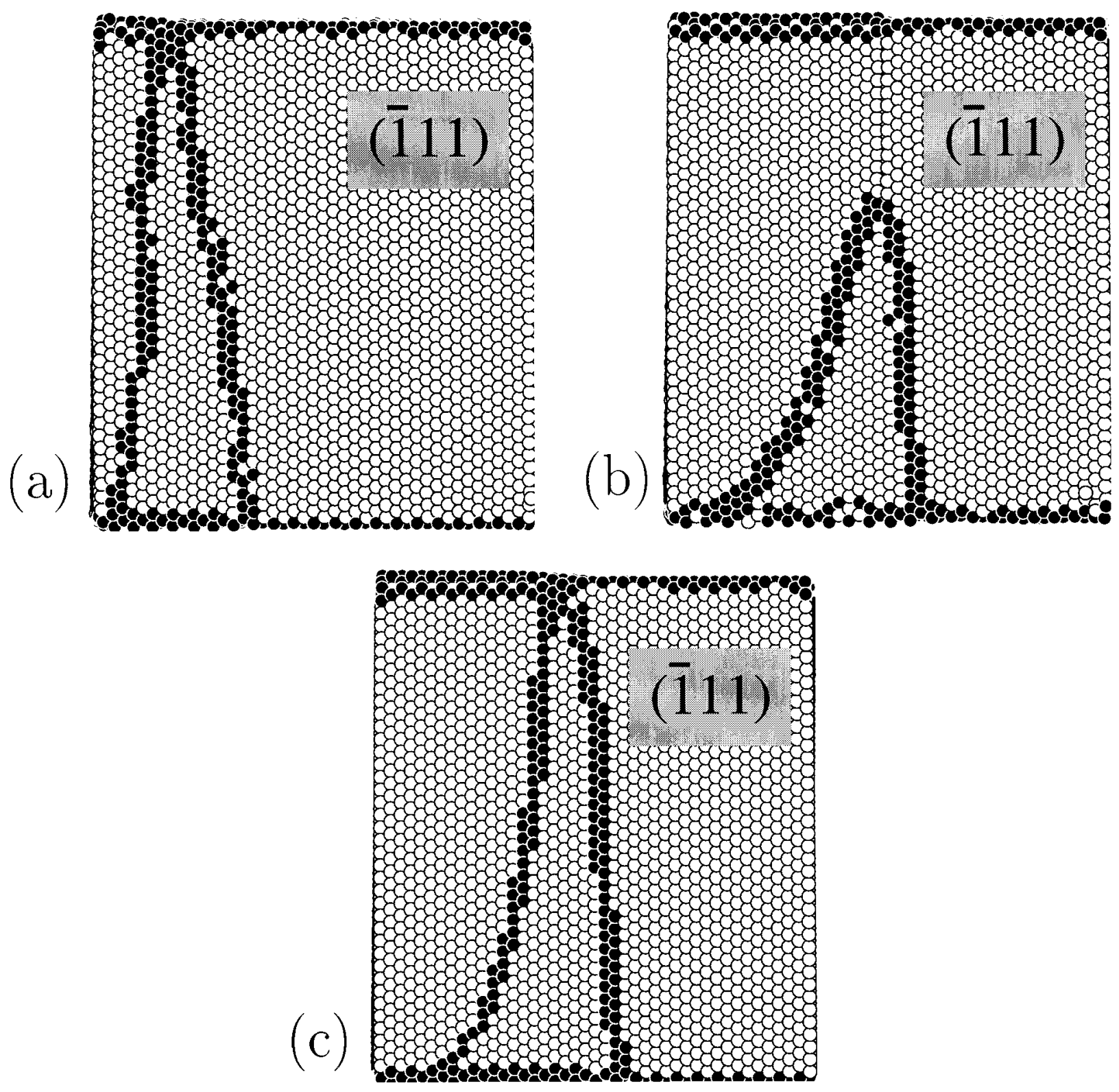

FIG. 9. Room-temperature simulations with two free (110) surfaces. (a) Partials initially in the ( $\overline{1} 11)$ plane. The partials recombine in the top of the crystal and are attracted to the free (1 $\overline{1} 1)$ surface. (b) Partials initially in the (1 $\overline{1} 1)$ plane. The dislocation has cross slipped from the $(1 \overline{1} 1)$ plane to the $(\overline{1} 11)$ plane in the bottom half of the crystal, thereby creating a screwlike constriction. The trace of the stacking fault between the partials of the non-cross-slipped part of the dislocation is seen in the upper half of the crystal. (c) Partials initially on the (1 $\overline{1} 1)$ plane. The cross slip is complete, and the dislocation is entirely in the $(\overline{1} 11)$ plane. The configuration has been quenched by MDmin minimization.

with the partials parallel to the step in both ends, when the step orientations have been switched, would result in creation of an edgelike constriction. The simulations with the step in the top parallel to the $(1 \overline{1} 1)$ plane and the step in the bottom parallel to the $(\overline{1} 11)$ plane showed the expected behavior. Both systems create a configuration with a screwlike constriction and the partials parallel to the step in either end. The system with the partials initially parallel in the $(1 \overline{1} 1)$ plane performs cross slip in the top of the crystal whereas the system with partials initially parallel in the (111) plane performs cross slip in the bottom of the crystal. For the two systems with the switched step orientation the results are different. The partials stay in their initial glide plane, adopting a configuration with a perfect screw dislocation in one end of the crystal and two nonparallel Shockley partials in the other end of the crystal. There are no signs of cross slip leading to configurations with an edgelike constriction and the partials parallel to the steps in either end.

These simulations demonstrate the influence of a free (110) surface on the configuration of a dissociated screw dislocation perpendicular to that surface. We have qualitatively confirmed the predictions by elasticity theory of the effect of a free surface. In addition we have observed a strong preference for the partials to be in the glide plane parallel to the surface step. 
(a)

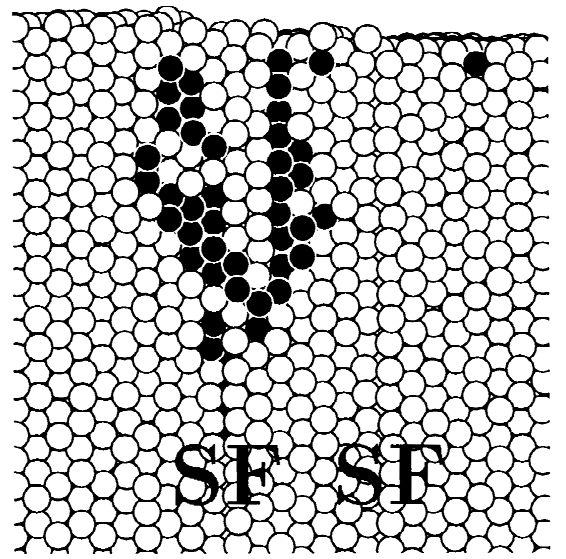

(b)

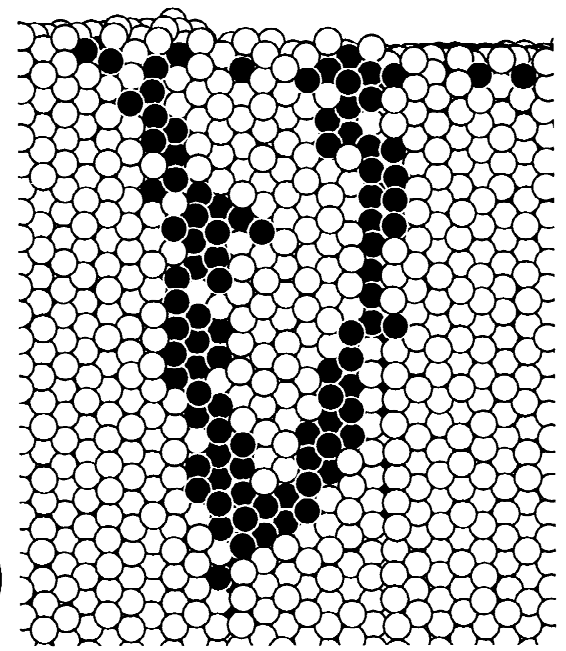

(c)

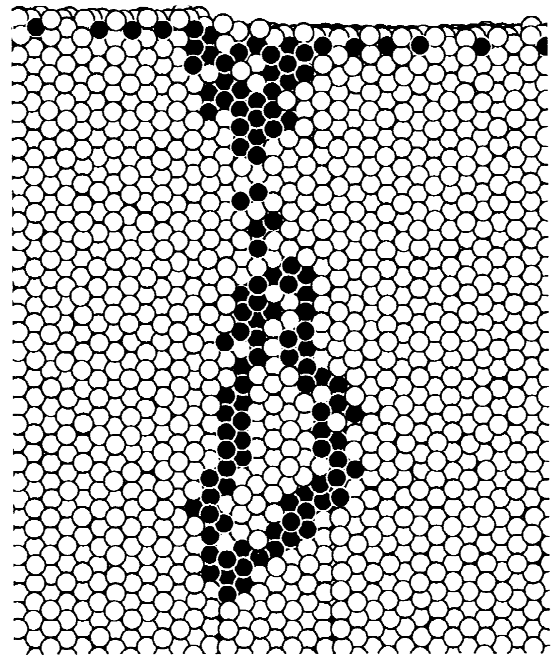

$(\mathrm{d})$

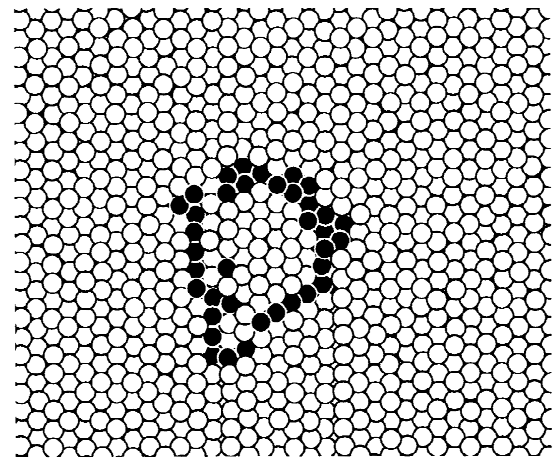

FIG. 10. Snapshots from the simulation showing annihilation of two screw dislocations of opposite signs. (a)-(d) (111) planes. (a) The dislocation with $b=\frac{1}{2}[\overline{11} 0]$ has performed a cross slip in the top of the crystal, thereby creating a screwlike constriction. The presence of the dislocation with $b=\frac{1}{2}[110]$ in a ( $\left.1 \overline{1} 1\right)$ plane and the non-cross-slipped part of the $b=\frac{1}{2}[\overline{11} 0]$ dislocation also in a (1 $\left.\overline{1} 1\right)$ plane is clearly seen as two vertical lines of stacking fault marked SF separated by seven $\{111\}$ plane spacings. (b) A new Shockley partial (vertical) has been created to the right in the ( $\overline{1} 11)$ plane. The Shockley partial to the left is the originally cross-slipped $\frac{1}{6}[\overline{12} 1]$ and the small inclined part connecting these two is the remains of the $\frac{1}{6}[\overline{211}]$ Shockley partial. (c) The newly created Shockley partial $\frac{1}{6}[12 \overline{1}]$ to the right reacts with the $\frac{1}{6}[\overline{12} 1]$ Shockley partial and they annihilate. (d) Annihilation in the top of the crystal, leaving a small stacking-fault loop comprising the $\frac{1}{6}[\overline{211}]$ and the $\frac{1}{6}[\overline{12} 1]$ Shockley partials. The stacking fault loop moves down through the crystal and disappears at the bottom, leaving the crystal defect free.

\section{Annihilation of two screw dislocations}

An interesting parameter which is believed to be closely related to cross slip is the minimum stable dipole height of screw dislocations of opposite signs. Experiments ${ }^{3}$ have shown this length to be $50-500 \mathrm{~nm}$ in $\mathrm{Cu}$. Screw dislocations of opposite signs in different glide planes closer than this minimum dipole height are believed to cross slip and annihilate. Due to the length and time scale, this problem is not suited for ordinary atomistic MD simulations. However, it might be possible to obtain insight into the cross-slip mechanism of a single screw dislocation, by performing MD simulations of systems containing two screw dislocations of opposite signs at very close range. In order to speed up the simulations, it was decided to use a high temperature $(580 \mathrm{~K})$ and rather small systems with $w=8.6 \mathrm{~nm}$ and $h=50 b(12.7$ $\mathrm{nm})$. The systems consisted of 76050 atoms. The two screw dislocations with Burgers vectors $\pm \frac{1}{2}[110]$ were initially introduced in the system as two perfect screw dislocations in different glide planes separated by only $\sim 2.2 \mathrm{~nm}$. We performed a simulation with periodic boundary conditions, along the $[110]$ direction and a simulation without periodic boundary conditions, i.e., with two free (110) surfaces.

The simulation with periodic boundary conditions showed no sign of cross slip. The two dislocations dissociate in two parallel (11 1) planes as in Figs. 4(a) and 4(c) separated by seven $\{111\}$ plane spacings, with splitting widths fluctuating around an average value of $\sim 1 \mathrm{~nm}$. The small value of the splitting width must be attributed to the presence of the other dislocation, because no significant temperature effect was observed in the simulations of just one dislocation in Sec. IV A 1. Occasionally the partials in the same glide plane were so close that the dislocation might be thought of as recombined. However, as mentioned for the partials in Sec. IV A 1, there is an in-plane smearing of the dislocation which confines it to a particular glide plane and inhibits cross slip. The simulation used 4600 time steps corresponding to 
$\sim 25$ ps. In the context of cross slip this is a very short simulation, and it is in no way possible to rule out the possibility of some kind of bulk cross slip on the basis of this simulation.

To investigate the role of free surfaces in this kind of system, we removed the periodic boundary conditions along the [110] direction from the above-mentioned system. This produced cross slip of one of the dislocations and led to annihilation of the two screw dislocations through successive dislocation reactions. It was possible to monitor the detailed dislocation reactions, thereby enabling exact specification of the different stages leading to the annihilation. When the periodic boundary conditions were removed, the two screw dislocations were split into Shockley partials in two parallel (1 $\overline{1} 1)$ planes separated by seven $\{111\}$ plane spacings. The annihilation of the two screw dislocations is initiated at the top of the crystal, and proceeds downwards through the crystal in the following manner. The dislocation with $b=\frac{1}{2}[\overline{110}]$ performs cross slip to the $(\overline{1} 11)$ plane in the top of the crystal, thereby creating a screwlike constriction on the dislocation; see Fig. 10(a). The Shockley partial $\frac{1}{6}[\overline{211}]$, glissile in the $(\overline{1} 11)$ plane, is attracted to the Shockley partial $\frac{1}{6}[121]$ glissile in the $(1 \overline{1} 1)$ plane, and the two partials react to produce a sessile stair-rod dislocation: ${ }^{1}$

$$
\frac{1}{6}[\overline{211}]_{(\overline{1} 11)}+\frac{1}{6}[121]_{(1 \overline{1} 1)} \rightarrow \frac{1}{6}[\overline{1} 10] .
$$

The stair-rod dislocation, not shown in Fig. 10, is located at the intersection of the $(1 \overline{1} 1)$ plane containing the $b=\frac{1}{2}[110]$ dislocation and the $(\overline{1} 11)$ plane containing the newly cross-slipped part of the $b=\frac{1}{2}[\overline{110}]$ dislocation. Hence, the stair-rod dislocation is in the same (111) plane as the $\frac{1}{6}[21 \overline{1}]$ Shockley partial [see, e.g., Fig. 4(a)] and attracts this:

$$
\frac{1}{6}[\overline{1} 10]+\frac{1}{6}[21 \overline{1}]_{(1 \overline{1} 1)} \rightarrow \frac{1}{6}[12 \overline{1}]_{(\overline{1} 11)} .
$$

The resultant Shockley partial $\frac{1}{6}[12 \overline{1}]$ is glissile in the same (111) plane as the cross-slipped part of the $b=\frac{1}{2}[\overline{110}]$ dislocation; see Figs. 10(b) and 10(c). The newly generated Shockley partial reacts with the remaining $\frac{1}{6}[\overline{12} 1]$ Shockley partial in the $(\overline{1} 11)$ plane, and the two partials annihilate:

$$
\frac{1}{6}[12 \overline{1}]_{(\overline{1} 11)}+\frac{1}{6}[\overline{12} 1]_{(\overline{1} 11)} \rightarrow 0 .
$$

The reactions create a stacking fault loop in the $(\overline{1} 11)$ plane which moves down through the crystal while the annihilation takes place; see Fig. 10(d). Eventually the loop reaches the bottom of the crystal and disappears, leaving the crystal defect free. Hence, once one of the dislocations has performed cross slip creating a screwlike constriction, there is no or a very low-energy barrier for the annihilation.

\section{CONCLUSION}

In this paper we have addressed the problem of cross slip in $\mathrm{Cu}$ with a purely atomistic method. The result for the minimum separation between noninteracting constrictions is in perfect agreement with results obtained from elasticity theory. Our result for the stress-free activation energy in the Friedel-Escaig cross-slip mechanism is in very good agreement with an earlier nonatomistic approach, ${ }^{5}$ but it is somewhat higher than other estimates ${ }^{10,6}$ derived from elasticity theory.

Our atomistic simulations also show that the two constrictions necessary in the Friedel-Escaig cross-slip mechanism are not equivalent, and that one of them, the screwlike constriction, is energetically favored compared to two parallel Shockley partials. The nonequivalence of the two constrictions is a fact not usually appreciated in the literature. The differences have been investigated, qualitatively and quantitatively.

As we have pointed out, the activation energies obtained from elasticity theory are subject to substantial uncertainty due to the break down of the isotropic elasticity relation describing the splitting of a perfect screw dislocation. Whether the overall quantitative agreement between the atomistic and the elastic approaches is an indication of a deeper concordance or merely fortuitous can only be resolved by detailed elastic work on isolated constrictions.

The effect of having the dislocation perpendicular to a free surface has been investigated, and surface-nucleated cross slip observed. The important feature in surfacenucleated cross slip is the possibility of creating a screwlike constriction without the accompanying edgelike constriction needed for bulk cross slip. On the other hand, we have also observed a strong preference for the partials to be in a glide plane parallel to the surface step, meaning that there is an energy barrier for cross slip into the glide plane not parallel to the surface step. Hence, more quantitative work on the energetics of different dislocation configurations close to a free surface needs to be done before we draw conclusions about the role of surface-nucleated cross slip.

In a simulation of two screw dislocations of opposite signs, surface-nucleated cross slip initiated the annihilation which proceeded via successive energetically favorable reactions. The atomistic approach allowed monitoring of the detailed dislocation reactions and thereby exact specification of the intermediate stages leading to the annihilation.

The role of cross slip in different macroscopic phenomena such as fatigue and plastic deformation is today well established. However, the present understanding of the intrinsic properties of cross slip is still rather nebulous. The results obtained in the present work may help to establish a better understanding. Altogether we may conclude that atomistic simulations are well suited for problems involving dislocation interactions at the nanoscale, where the use of methods based on elasticity theory is questionable.

\section{ACKNOWLEDGMENTS}

We are grateful to J. Bilde-Sørensen for drawing our attention to the experiments involving free surfaces, and we thank Jakob Schiøtz for providing the code for the dislocation finding program. We also thank Jim Sethna for helpful discussions. Center for Atomic-scale Materials Physics (CAMP) is sponsored by the Danish National Research 
Foundation. The present work was in part financed by The Danish Research Councils through Grant No. 9501775 and was done as a collaboration between CAMP and the Engineering Science Center for Structural Characterization and
Modelling of Materials at the Materials Research Department, Ris $\phi$. K.W.J. also acknowledges support from the U.S. Department of Energy through Grant No. DEFG0288ER45364.
${ }^{1}$ J.P. Hirth and J. Lothe, Theory of Dislocations, 2nd. ed. (Krieger, Malabar, 1992).

${ }^{2}$ L. M. Brown, Metall. Trans. A 22, 1693 (1991).

${ }^{3}$ U. Essmann and H. Mughrabi, Philos. Mag. A 40, 731 (1979).

${ }^{4}$ O. B. Pedersen, Philos. Mag. A 73, 829 (1996).

${ }^{5}$ M. S. Duesbery, N. P. Louat, and K. Sadananda, Acta Metall. Mater. 40, 149 (1992).

${ }^{6}$ W. Püschl, and G. Schoeck, Mater. Sci. Eng. A 164, 286 (1993).

${ }^{7}$ W. Jones and N. H. March, Theoretical Solid State Physics (Wiley, New York, 1973), Vol. 1.

${ }^{8}$ K. W. Jacobsen, P. Stoltze, and J. K. Nørskov, Surf. Sci. 366, 394 (1996), and references therein; K. W. Jacobsen J. K. Nørskov, and M. J. Puska, Phys. Rev. B 35, 7423 (1987).

${ }^{9} \mathrm{~J}$. Friedel, in Dislocations and Mechanical Properties of Crystals, edited by J.C. Fisher (Wiley, New York, 1957).

${ }^{10}$ B. Escaig, in Dislocation Dynamics, edited by A.R. Rosenfeld, G.T. Hahn, A.L. Bement, Jr., and R.I. Jaffee, McGraw-Hill Series in Materials-Science and Engineering (McGraw-Hill, New York, 1968); B. Escaig, J. Phys. (France) 29, 225 (1968).

${ }^{11}$ R. Fournet and J. M. Salazar, Phys. Rev. B 53, 6238 (1996), and references therein.

${ }^{12}$ B. Devincre, in Computer Simulation in Materials Science (Nano/ Meso/Macroscopic Space \& Time Scales), edited by H.O. Kirchner, L.P. Kubin, and V. Pontikis (Kluwer Academic, Dordrecht, 1996).

${ }^{13}$ E. Aernoudt, P. Van Houtte, and T. Leffers, Materials Science and Technology (VCH, Weinheim, 1993), Vol. 6.

${ }^{14}$ O. B. Pedersen and A. T. Winter, Phys. Status Solidi A 149, 281 (1995).

${ }^{15}$ G. Schoeck and A. Seeger, Report on the Conference of Defects in Crystalline Solids, Bristol, 1954 (The Physical Society, London, 1955), p. 340.

${ }^{16}$ R. L. Fleischer, Acta Metall. 7, 134 (1959).

${ }^{17}$ P. B. Hirsch, Philos. Mag. 7, 67 (1962).

${ }^{18}$ A. N. Stroh, Proc. Phys. Soc. London, Sec. B 67, 427 (1954).

${ }^{19}$ J. Bonneville and B. Escaig, Acta Metall. 27, 1477 (1979).
${ }^{20}$ J. Bonneville, B. Escaig, and J. L. Martin, Acta Metall. 36, 1989 (1988).

${ }^{21}$ H. -J. Möller, H. Ewaldt, and P. Haasen, Phys. Status Solidi A 55, 469 (1979)

${ }^{22}$ F. Minari, B. Pichaud, and L. Capella, Philos. Mag. 31, 275 (1975).

${ }^{23}$ P. M. Hazzledine, H. P. Karnthaler, and E. M. Winther, Philos. Mag. 32, 81 (1975); E. Winther, H. P. Karnthaler, and P. M. Hazzledine, Proceedings of the 4th International Conference on the Strength of Metals and Alloys (Laboratoire de Physique du Solide, Nancy, 1976), Vol. 2.

${ }^{24}$ M. S. Duesbery, N. P. Louat, and K. Sadananda, Philos. Mag. A 65, 311 (1992).

${ }^{25}$ G. Saada, Mater. Sci. Eng. A 137, 177 (1991).

${ }^{26}$ W. M. Stobbs and C. H. Sworn, Philos. Mag. 24, 1365 (1971).

${ }^{27}$ J. Schiøtz, K. W. Jacobsen, and O. H. Nielsen, Philos. Mag. Lett. 72, 245 (1995).

${ }^{28}$ J. Schiøtz, T. Rasmussen, K. W. Jacobsen, and O. H. Nielsen, Philos. Mag. Lett. 74, 339 (1996).

${ }^{29}$ M. R. Sørensen, K. W. Jacobsen, and P. Stoltze, Phys. Rev. B 53, 2101 (1996).

${ }^{30}$ S. Crampin, K. Hampel, D. D. Vvedensky, and J. M. MacLaren, J. Mater. Res. 5, 2107 (1990).

${ }^{31}$ K. Stokbro and K. W. Jacobsen, Phys. Rev. B 47, 4916 (1993).

${ }^{32}$ J. Schiøtz, Ph.D thesis, Technical University of Denmark, 1995.

${ }^{33}$ P. Stoltze, Simulation Methods in Atomic-scale Materials Physics (Polyteknisk Forlag, Lyngby, 1997).

${ }^{34}$ H. Häkkinen, S. Mäkinen, and M. Manninen, Phys. Rev. B 41, 12441 (1990).

${ }^{35}$ J. Huang, M. Meyer, and V. Pontikis, Phys. Rev. B 42, 5495 (1990).

${ }^{36}$ R. C. Perrin and E. J. Savino, J. Microsc. 98, 214 (1973).

${ }^{37}$ D. J. Bacon, D. M. Barnett, and R. O. Scattergood, Prog. Mater. Sci. 23, 51 (1978)

${ }^{38}$ C. Kittel, Introduction to Solid State Physics, 4th. ed. (Wiley, New York, 1971). 